\title{
Impact of treatment modality on long-term survival of stage IA small-cell lung cancer patients: a cohort study of the U.S. SEER database
}

\author{
Shao-Feng Lin ${ }^{1 \#}$, Yu-Zhen Zheng ${ }^{2 \#}$, Xiao-Qiang Li $^{3 \#}$, Hai-Peng Xu ${ }^{4}$, Jun-Jie Wang ${ }^{5}$, Wei Wang ${ }^{6}$, \\ Qing-Yuan Huang ${ }^{7}, \mathrm{Da} \mathrm{Wu}^{3}$, Chen-Xi Zhong ${ }^{8}$, Shen-Shen Fu' ${ }^{9}$, Lian-Xiong Yuan ${ }^{10}$, Si-Chao Wang ${ }^{11}$, \\ Rui-Xing Luo ${ }^{3}$, Wen-Yu Zhai ${ }^{12}$, Ben-Tong Yu ${ }^{13}$, Kun-Shou Zhu ${ }^{1}$ \\ ${ }^{1}$ Department of Thoracic Surgery, Fujian Cancer Hospital \& Fujian Medical University Cancer Hospital\& Fujian Provincial Key Laboratory of \\ Tumor Biotherapy, Fuzhou, China; ${ }^{2}$ Department of Thoracic Surgery, The Sixth Affiliated Hospital, Sun Yat-sen University, Guangzhou, China; \\ ${ }^{3}$ Department of Thoracic Surgery, Peking University Shenzhen Hospital, Shenzhen, China; ${ }^{4}$ Department of Thoracic Oncology, Fujian Cancer \\ Hospital \& Fujian Medical University Cancer Hospital, Fuzhou, China; ${ }^{5}$ Department of Surgery, Cleveland Clinic, Cleveland, Ohio, USA; ${ }^{6}$ S. \\ Arthur Localio Laboratory, Department of Surgery, New York University School of Medicine, New York, NY, USA; ${ }^{7}$ Department of Thoracic \\ Surgery, Shanghai First People's Hospital, Shanghai Jiao Tong University, Shanghai, China; ${ }^{8}$ Department of Thoracic Surgery, Shanghai Chest \\ Hospital, Shanghai Jiao Tong University, Shanghai, China; ' Department of Ultrasonography, Guangzhou First People's Hospital, Guangzhou \\ Medical University, Guangzhou, China; ${ }^{10}$ Office of Research Service, Third Affiliated Hospital of Sun Yat-sen University, Guangzhou, Guangzhou, \\ China; ${ }^{11}$ Department of Thoracic Surgery, Guangdong Provincial People's Hospital, Guangzhou, China; ${ }^{12}$ Department of Thoracic Oncology, Sun \\ Yat-sen University Cancer Center, State Key Laboratory of Oncology in South China, Collaborative Innovation, Guangzhou, China; ${ }^{13}$ Department \\ of Thoracic Surgery, First Affiliated Hospital of Nanchang University, Nanchang, China \\ Contributions: (I) Conception and design: SF Lin, XQ Li, YZ Zheng, KS Zhu, BT Yu; (II) Administrative support: KS Zhu, BT Yu; (III) Provision \\ of study materials or patients: HP Xu, JJ Wang, W Wang, QY Huang; (IV) Collection and assembly of data: D Wu, CX Zhong, SS Fu, LX Yuan; \\ (V) Data analysis and interpretation: SF Lin, YZ Zheng, SC Wang, RX Luo, WY Zhai; (VI) Manuscript writing: All authors; (VII) Final approval of \\ manuscript: All authors. \\ \#These authors contributed equally to this study. \\ Correspondence to: Professor Kun-Shou Zhu, MD. Department of Thoracic Oncology, Fujian Cancer Hospital \& Fujian Medical University Cancer \\ Hospital, Fuzhou, China. Email: zhuks@fjzlhospital.com; Professor Ben-Tong Yu. Department of Thoracic Surgery, First Affiliated Hospital of \\ Nanchang University, Nanchang, China. Email: yubentongcdyfy@163.com.
}

Background: The optimal treatment modality for patients with stage IA (T1N0M0) small-cell lung cancer (SCLC) is still unclear.

Methods: Patients who received surgical resection or chemo-radiotherapy (CRT) between January 2004 and December 2014 were identified from The Surveillance, Epidemiology and End Results (SEER) database. Surgical resection included lobectomy, wedge resection, segmentectomy with lymphadenectomy [examined lymph node $(E L N) \geq 1$. Propensity score match analysis was utilized to balance the baseline characteristics.

Results: A total of 686 stage IA SCLC cases were included: 337 patients underwent surgery and 349 patients were treated by CRT alone. Surgery achieved a better outcome than CRT alone, with an adjusted hazard ratio (HR) of 0.495 . Patients who underwent lobectomy demonstrated a longer overall survival (OS), compared to those who received sublobectomy (crude cohort, median OS, 69 vs. 38 months; match cohort, median OS, 67 vs. 38 months). Patients with ELN $>7$ presented with longer OS than those with ELN $\leq 7$ (crude cohort, median OS, 91 vs. 49 months; matched cohort, median OS, 91 vs. 54 months). The additional efficacy of chemotherapy or radiotherapy in patients receiving lobectomy was observed. The best prognosis was achieved in the lobectomy plus CRT cohort, with a 5-year survival rate of $73.5 \%$.

Conclusions: The prolonged survival associated with lobectomy and chemotherapy or radiotherapy presents a viable treatment option in the management of patients with stage IA SCLC.

Keywords: Small-cell lung cancer (SCLC); SEER; treatment; early-stage lung cancer 
Submitted Jun 08, 2020. Accepted for publication Oct 19, 2020.

doi: $10.21037 /$ atm-20-5525

View this article at: http://dx.doi.org/10.21037/atm-20-5525

\section{Introduction}

Lung cancer is the leading cause of cancer incidence and mortality, with 2.1 million new lung cancer patients being diagnosed in 2018 around the world (1). Small-cell lung cancer (SCLC) constitutes 13\% of all lung cancers and is characterized by a rapid doubling time, higher rate of relapse, and shorter survival (2). Up to $70 \%$ of SCLC patients have metastases at the time of diagnoses (3). Treatment advances for SCLC have been limited over the past few decades, with many patients facing a high likelihood of poor prognoses. Currently, chemotherapy and radiotherapy remain the mainstay management for most SCLC patients (4). In recent years, several studies have established a correlation between surgical intervention for early stage SCLC and improved outcomes (5-9). However, the details identifying the most effective resection type and required number of lymph nodes for assessment to achieve improved outcomes have not been determined. In addition, lobectomy has long been regarded indispensable in the management of operable lung cancer, whereas sublobectomy is traditionally introduced when the pulmonary function cannot tolerate lobectomy $(10,11)$. Recent studies indicate that, the prognosis is fairly well in nature for nonsmall cell lung cancer (NSCLC) with T1NOM0 stage, and sublobar resection would achieve comparable prognosis to lobectomy in this cohort $(12,13)$. We speculate that, same association might also exist in SCLC with T1N0M0 stage. Furthermore, the benefit of additional chemotherapy or radiotherapy for patients with resected SCLC remains unclear. To identify an appropriate treatment program for stage IA SCLC patients, the prognosis of this patient population who underwent surgery or chemo-radiotherapy (CRT) was examined. We present the following article in accordance with the STROBE reporting checklist (available at http://dx.doi.org/10.21037/atm-20-5525).

\section{Methods}

\section{Patient population}

The study was conducted in accordance with the Declaration of Helsinki (as revised in 2013) (14). Patients diagnosed with SCLC between January 2004 and December
2014 were identified from the SEER database. The SEER Program of the National Cancer Institute is responsible for the collection and reporting of cancer incidence and survival data from several population-based central cancer registries, covering approximately $30 \%$ of the U.S. population. The SEER-18 registries database was queried for all patients using SEER*Stat version 8.3.2.

The inclusion criteria included the following: (I) histologically confirmed SCLC; (II) receiving CRT or surgery (lobectomy, wedge resection, or segmentectomy) as the initial treatment; (III) IA stage (T1N0M0 status) according to the $8^{\text {th }}$ edition tumor-node-metastasis (TNM) staging system. For patients who underwent surgery, lymphadenectomy with at least one examined lymph node (ELN) was required. Variables on demographics, stage of disease, tumor diameter, invasion scope, nodal status, distant metastasis, and survival were collected. Patients without documented variables, or diagnosed with SCLC from an autopsy or death certificate were excluded. Patient staging was performed in accordance with the American Joint Committee on Cancer (AJCC) $8^{\text {th }}$ edition TNM staging system (15).

\section{Statistics}

The survival rate was calculated using the Kaplan-Meier method. Categorical variables were tabulated by frequency and percentage. Correlations among clinicopathological characteristics were assessed with Chi-squared testing. To identity the prognostic impact of resection type (lobectomy $v s$. sublobectomy) and ELN ( $\leq 7 v s .>7)$, propensity score matching (PSM) was conducted as described by Rosenbaum and Rubin $(16,17)$. The propensity score for an individual was calculated on concomitant variables with potential prognostic significance (18). The concomitant variables in the PSM analysis for resection type (lobectomy $v s$. sublobectomy) and ELN ( $\leq 7 v s .>7)$ included age, gender, radiotherapy, and chemotherapy. Resection type was included as a concomitant variable in the PSM analysis for ELN. Additionally, ELN was an additional concomitant variable in the PSM analysis for resection type. Kernel density estimates indicated the distribution of propensity scores in each group (19), while univariate and multivariate 
Table 1 Characteristics of stage IA SCLC patients

\begin{tabular}{|c|c|c|c|c|c|c|}
\hline Variables & Case & 5 -year OS (\%) & $\mathrm{P}^{\mathrm{a}}$ & \multicolumn{3}{|c|}{ Multivariate analysis } \\
\hline \multicolumn{7}{|l|}{ Age (years) } \\
\hline$\leq 65$ & $221(32.2)$ & 49.5 & $<0.001$ & Reference & & $<0.001$ \\
\hline$>65$ & 465 (67.8) & 31.1 & & 1.656 & $1.312-2.089$ & \\
\hline Male & $312(45.5)$ & 34.7 & 0.060 & Reference & & 0.209 \\
\hline Female & $374(54.5)$ & 39.1 & & 0.878 & $0.716-1.076$ & \\
\hline \multicolumn{7}{|l|}{ Location } \\
\hline Upper & $430(62.7)$ & 36.9 & 0.984 & & & \\
\hline \multicolumn{7}{|l|}{ Lateral } \\
\hline Left & $282(41.1)$ & 39.6 & 0.851 & & & \\
\hline Right & 404 (58.9) & 35.5 & & & & \\
\hline \multicolumn{7}{|l|}{ T status } \\
\hline $\mathrm{T} 1 \mathrm{a}$ & $68(9.9)$ & 47.3 & 0.039 & Reference & & 0.213 \\
\hline $\mathrm{T} 1 \mathrm{~b}$ & $327(47.7)$ & 34.4 & & 1.385 & $0.925-2.074$ & \\
\hline $\mathrm{T} 1 \mathrm{c}$ & $291(42.4)$ & 39.3 & & 1.229 & $0.813-1.856$ & \\
\hline \multicolumn{7}{|l|}{ Therapy } \\
\hline
\end{tabular}

a , univariate Cox analysis; ${ }^{b}$, multivariate Cox analysis. SCLC, small-cell lung cancer; OS, overall survival; HR, hazard ratio; Cl, confidence interval; CRT, chemoradiotherapy.

analysis were performed to identify the independent prognostic factors. Multivariate analysis was used for factors that demonstrated statistical significance $(\mathrm{P}<0.1)$ in univariate analysis. Statistical significance was assumed at a two-sided probability value $<0.05$. All statistical analyses were performed with the SPSS 22.0 (IBM Corp., NY, USA) and $\mathrm{R}$ (version 3.3.2) software packages.

\section{Results}

\section{Patient characteristics}

A total of 686 stage IASCLC patients with were included in this study. Surgery was performed in 337 patients (49.1\%) with the remaining 349 patients $(50.9 \%)$ treated by CRT alone. The median age was 68 years (range, 43 to 89 years). For status, 68, 327, and 291 patients were classified with T1a, T1b, and T1c status, respectively. The median tumor size was $17 \mathrm{~mm}$. Patient characteristics are listed in Table 1 .

Lobectomy, wedge resection, and segmentectomy were performed in 247 (73.3\%), 71 (21.1\%), and $19(5.6 \%)$ of patients who underwent surgery, respectively. The median ELN was 7 (ranging between 1 and 87). In patients with resected SCLC, additional radiotherapy and chemotherapy were performed in 74 patients $(22.0 \%)$ and 189 patients (56.1\%), respectively. Oligo-modality (surgery alone), bi-modality (surgery plus chemo/radiotherapy), and trimodality (surgery plus CRT) therapies were applied in 146, 119 , and 72 patients, respectively. The treatment details of patients with resected SCLC are listed in Table S1. 


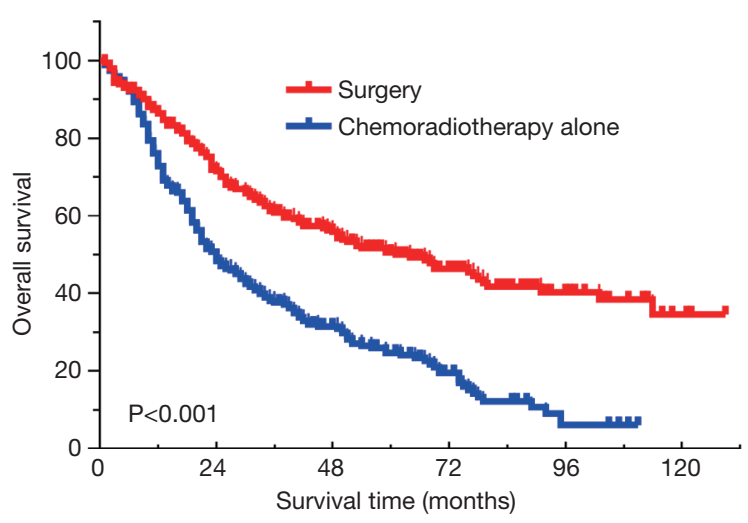

Figure 1 Surgery lead to better outcome compared to CRT alone in stage IA SCLC. CRT, chemoradiotherapy; SCLC, small-cell lung cancer.

\section{Surgery improved outcomes compared to CRT alone}

The median OS for the entire cohort was 35 months. The 5 -year survival rate for patients who underwent surgery and CRT alone was $50.0 \%$ and $24.7 \%$, respectively $(\mathrm{P}<0.001)$ (Figure 1). The superiority of surgery was further confirmed in multivariate analysis, with the adjusted hazard ratio (HR) of 0.495 [95\% confidence interval (CI), 0.401-0.611] (Table 1). Age was identified as the other independent prognostic factor.

For patients with resected SCLC, the 5-year survival rate for tri-modality, bi-modality, and oligo-modality therapy was $66.9 \%, 46.0 \%$, and $43.8 \%$, respectively (Figure S1). Tri-modality led to a better outcome than surgery alone (adjusted HR, 0.543; 95\% CI, 0.331-0.889) and bi-modality (adjusted HR, 0.641; 95\% CI, 0.389-1.057).

For patients with resected SCLC, lobectomy demonstrated an improved outcome compared to sublobectomy (median OS, 69 vs. 38 months, $\mathrm{P}=0.051$; Figure $2 A$ ). However, after PSM analysis, the superiority of lobectomy on survival was not significant in the matched cohort $(\mathrm{n}=188)$ (median OS, 67 vs. 38 months, $\mathrm{P}=0.223$; Figure $2 B$ ). Distribution of propensity-scores indicated balanced basic characteristics after PSM analysis (Figure 2C,D).

Resected SCLC patients with ELN $>7$ was associated with a better outcome (median OS, 91 vs. 49 months, $\mathrm{P}=0.038$; Figure 2E). However, following PSM analysis, the superiority of ELN $>7$ on survival was not significant in the matched cohort ( $\mathrm{n}=304)$ (median OS, 91 vs. 54 months, $\mathrm{P}=0.105$; Figure $2 F$ ). The distribution of the propensityscore indicated balanced basic characteristics after PSM analysis (Figure 2G,H).
To evaluate the interaction between surgery and additional treatment modalities in resected SCLC, univariate analysis was performed in oligo-modality, bimodality, and tri-modality cohorts. Compared with sublobectomy, the HR for lobectomy was 1.058, 0.569, and 0.385 in the oligo-modality, bi-modality, and tri-modality cohorts, respectively; compared with ELN $\leq 7$, the HR for ELN $>7$ was $0.704,0.660$, and 0.722 in the oligo-modality, bi-modality, and tri-modality cohorts, respectively. There seems to be asynergistic interaction between surgery and additional chemo/radiotherapy (Table 2).

\section{Surgery with CRT was the optimal treatment for stage $I A$ SCLC patients}

To determine the most effective therapeutic option, SCLC patients who underwent surgery were divided into the following groups, according to their treatment: sublobectomy ( $\mathrm{n}=38)$, lobectomy $(\mathrm{n}=108)$, sublobectomy plus chemo/radiotherapy $(\mathrm{n}=30)$, lobectomy plus chemo/ radiotherapy $(\mathrm{n}=89)$, sublobectomy plus CRT $(\mathrm{n}=22)$, and lobectomy plus CRT ( $\mathrm{n}=50$ ) (Table 3). As shown in Figure 3, patients who received lobectomy plus CRT showed the best prognosis, with the estimated 5 -year OS rate of $73.5 \%$. The adjusted HR for lobectomy alone, lobectomy plus chemo/radiotherapy, and lobectomy plus CRT was 1.095 , 0.851 , and 0.438 , respectively $(\mathrm{P}=0.012)$, suggesting an enhanced survival benefit from additional chemotherapy or radiotherapy for patients who undergo lobectomy. This phenomenon was not observed in patients who received sublobectomy. The adjusted HR for sublobectomy alone, sublobectomy plus chemo/radiotherapy, and sublobectomy plus chemo-radiotherapy was $1.000,1.304$, and 1.220 , respectively $(\mathrm{P}=0.752)$.

\section{Discussion}

In this study, we investigated the prognosis of patients with stage IA SCLC and found lobectomy plus CRT to be the optimal treatment program which provides the best prognosis.

The efficacy of surgery in limited staged SCLC has been reported by several studies over the past few decades (5-8). In this study, patients who underwent surgery showed significantly longer survival than those treated with CRT alone, with the median OS of 59 and 24 months, respectively. In addition, Zeng et al. included stage IA SCLC patients and compared the long-term survival between 

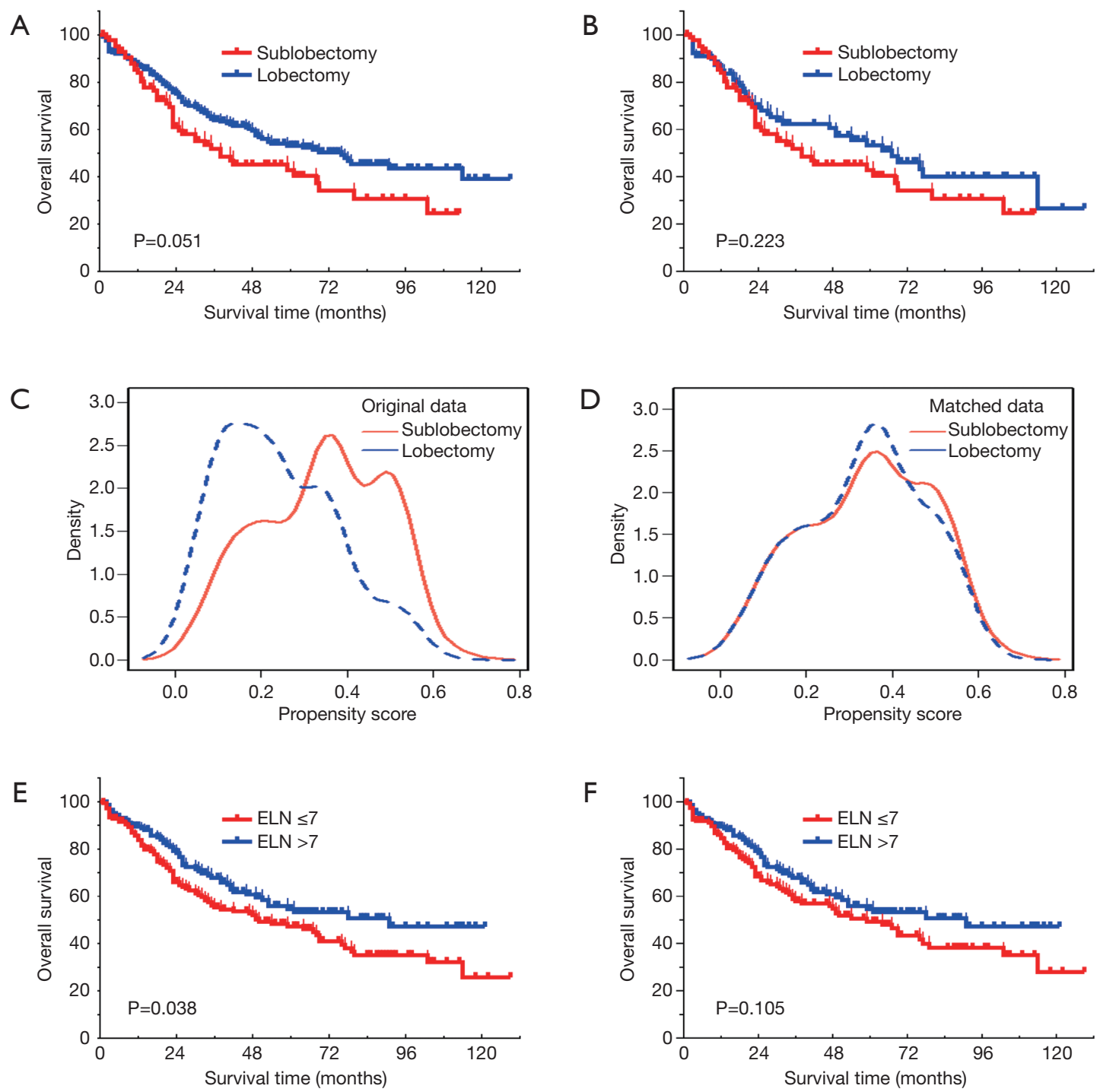

$\mathrm{F}$
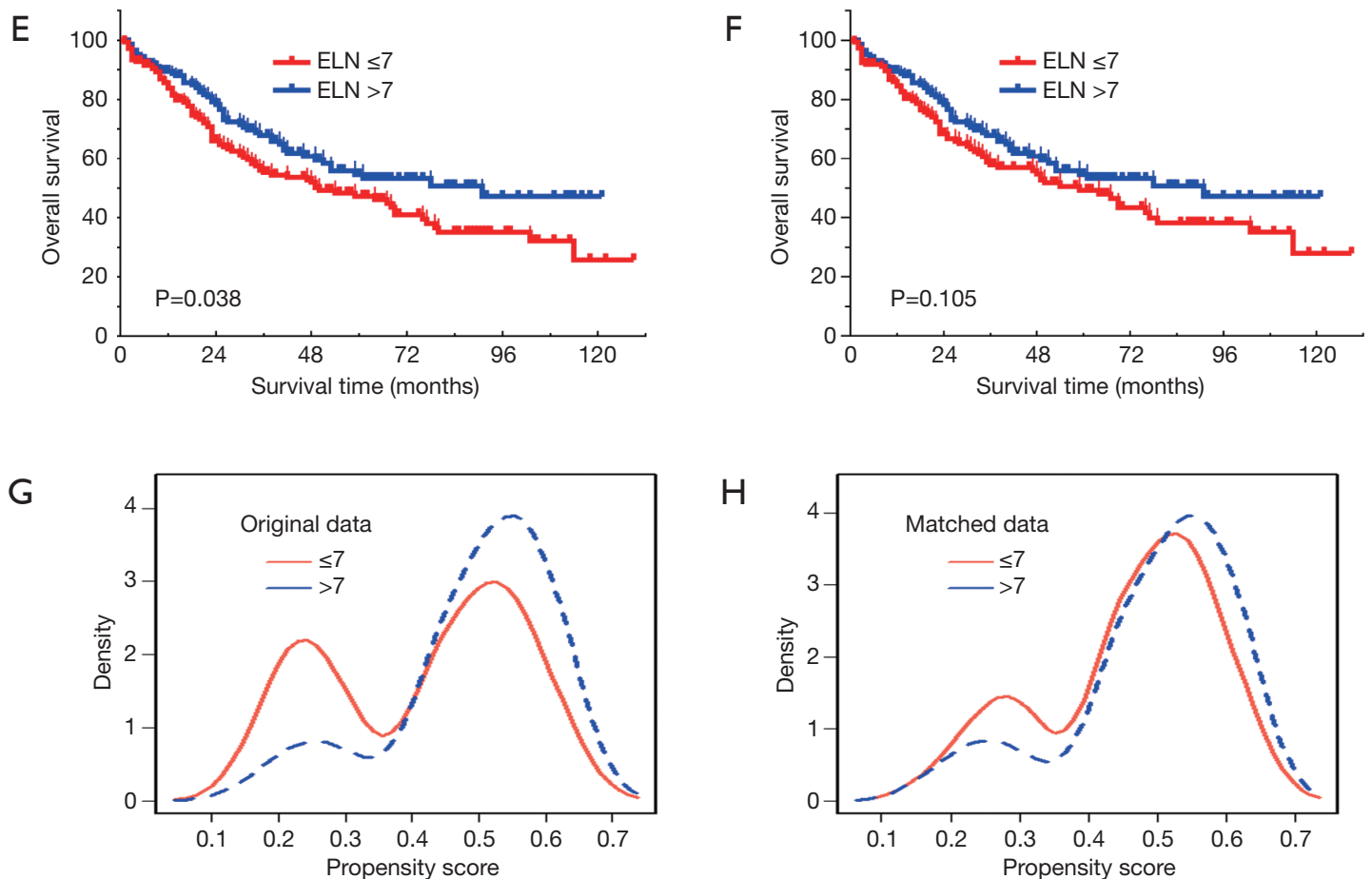

Figure 2 Comparison of OS between sublobectomy and lobectomy in the crude cohort (A) and matched cohort (B) of resected SCLC; distribution of propensity score of the crude cohort (C) and matched cohort (D) between sublobectomy and lobectomy in patients with resected SCLC; comparison of OS between ELNs $\leq 7$ and ELN $>7$ in the crude cohort (E) and matched cohort (F) of resected SCLC; distribution of propensity score of the crude cohort $(\mathrm{G})$ and matched cohort $(\mathrm{H})$ between ELN $\leq 7$ and ELN $>7$ in patients with resected SCLC.SCLC, small-cell lung cancer; ELN, examined lymph node. 
Table 2 Interaction between surgical parameters and treatment modalities in stage T1N0M0 SCLC

\begin{tabular}{|c|c|c|c|c|c|c|c|c|c|}
\hline Variables & \multicolumn{3}{|c|}{ Oligo-modality cohort $(n=146)$} & \multicolumn{3}{|c|}{ Bi-modality cohort $(n=119)$} & \multicolumn{3}{|c|}{ Tri-modality cohort $(\mathrm{n}=72)$} \\
\hline \multicolumn{10}{|l|}{ Resection type } \\
\hline Sublobectomy & Reference & & & Reference & & & Reference & & \\
\hline Lobectomy & 1.058 & $0.640-1.751$ & 0.826 & 0.569 & $0.315-1.027$ & 0.061 & 0.385 & $0.163-0.909$ & 0.030 \\
\hline$\leq 7$ & Reference & & & Reference & & & Reference & & \\
\hline$>7$ & 0.704 & $0.436-1.136$ & 0.151 & 0.660 & $0.383-1.136$ & 0.134 & 0.722 & $0.365-2.010$ & 0.857 \\
\hline
\end{tabular}

a, univariate Cox analysis. SCLC, small-cell lung cancer; ELN, examined lymph nodes; HR, hazard ratio; Cl, confidence interval.

Table 3 Impact of treatment on overall survival in patients with resected SCLC with T1N0M0 status

\begin{tabular}{|c|c|c|c|c|c|c|c|c|}
\hline Treatment group & \multicolumn{3}{|c|}{ Univariate analysis } & $\mathrm{P}_{\text {trend }}{ }^{\mathrm{a}}$ & \multicolumn{3}{|c|}{ Multivariate analysis } & $P_{\text {trend }}{ }^{b}$ \\
\hline Sublobectomy & Reference & & & 0.011 & Reference & & & 0.047 \\
\hline Lobectomy & 1.060 & $0.641-1.752$ & 0.822 & & 1.095 & $0.662-1.811$ & 0.725 & \\
\hline Sublob + chemo/radio & 1.274 & $0.664-2.443$ & 0.467 & & 1.304 & $0.680-2.501$ & 0.425 & \\
\hline Sublob + chemo + radio & 1.049 & $0.480-2.293$ & 0.904 & & 1.220 & $0.554-2.687$ & 0.621 & \\
\hline Lob + chemo + radio & 0.377 & $0.189-0.754$ & 0.006 & & 0.438 & $0.217-0.882$ & 0.021 & \\
\hline
\end{tabular}

${ }^{a}$, univariate Cox analysis; ${ }^{b}$, multivariate Cox analysis. SCLC, small cell lung cancer; HR, hazard ratio; Cl, confidence interval; sublob + chemo/radio, sublobectomy + chemotherapy/radiotherapy; lob + chemo/radio, lobectomy + chemotherapy/radiotherapy; sublob + chemo + radio, sublobectomy + chemotherapy + radiotherapy; lob + chemo + radio, lobectomy + chemotherapy + radiotherapy.

lobectomy and sublobar resection (20). In that study, patients who underwent chemoradiotherapy (CRT) has not yet been recruited. As is well known, CRT is the mainstay treatment for limited stage SCLC. Therefore, although better prognosis was found in patients who received lobectomy, it is still uncertain that it is the optimal treatment for SCLC with IA stage. Our result is consistent with the National Comprehensive Cancer Network (NCCN) guideline, which recommends surgical intervention for patients with stage I (T1-2N0M0) SCLC (21). The highlight of our study is that, patients with IA stage SCLC who underwent CRT and surgery and multimodality treatment were all included in this study. It is plausible that the study design might help to identify the optimal treatment in this cohort.

Lobectomy has long been considered as the standard surgical option for lung cancer. Goldstein et al. observed residual tumors in $45 \%$ of lobectomy specimens from
31 stage IA lung cancer patients, diagnosed by wedge resection (22). Lobectomy has been repeatedly established as superior to sublobectomy in providing better outcomes in most operable non-small cell lung cancer (NSCLC) patients $(11,23,24)$. Recently, sublobectomy was indicated to deliver prognoses comparable to lobectomy in stage IA NSCLC $(12,25)$. In this study, the PSM analysis showed no significance between lobectomy and sublobectomy on long-term survival $(\mathrm{P}=0.223)$. However, lobectomy is still recommended for two reasons. Firstly, the absolute risk was reduced in the lobectomy group (crude cohort: HR $=0.710,95 \%$ CI, 0.502-1.006; matched cohort: HR $=0.771,95 \%$ CI, 0.506-1.177). The survival duration was numerically longer for the lobectomy group (crude cohort, 69 vs. 38 months; matched cohort, 67 vs. 38 months), and the superiority of lobectomy was easily observed according to the survival curve. We speculate that the insignificant survival benefit among patients that received lobectomy 


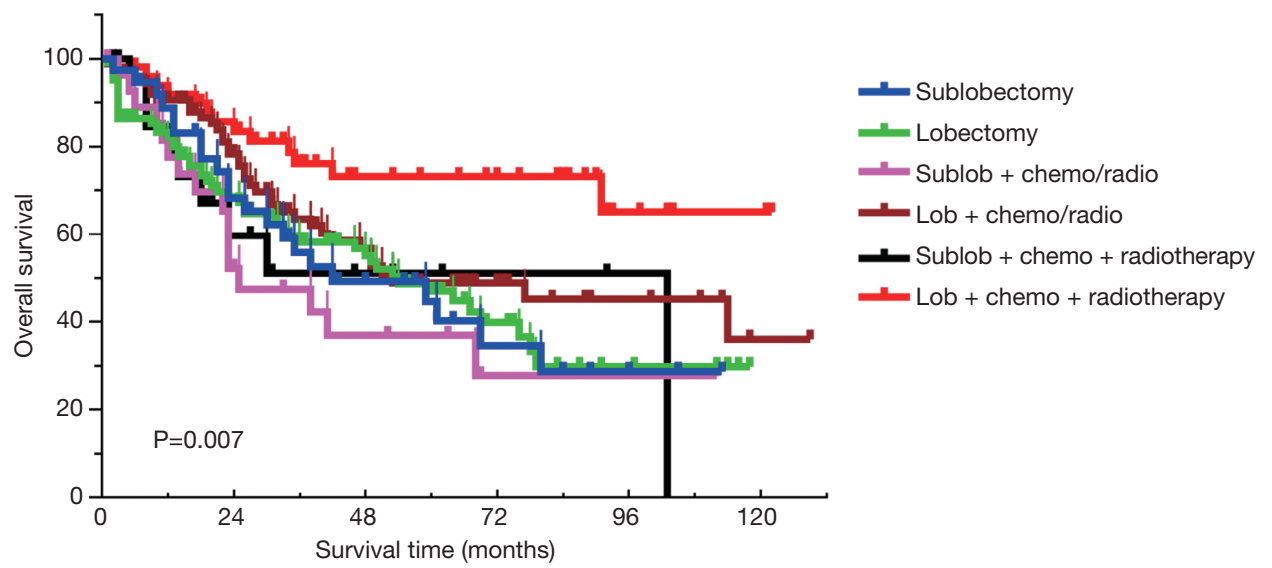

Figure 3 Comparison among different treatment modalities in patients with resected SCLC. SCLC, small-cell lung cancer; Sublob, sublobectomy; lob, lobectomy; chemo/radio, chemo/radiotherapy; chemo + radiotherapy, CRT.

is probably attributable to the limited sample size of the matched cohort $(n=188)$. Secondly, the synergistic effect of additional chemo/radiotherapy was only observed in patients who underwent lobectomy.

In this study, tri-modality (surgery plus CRT)treatment demonstrated significantly better outcomes for patients with resected stage IA SCLC compared with bi-modality (surgery plus chemo/radiotherapy) and oligo-modality (surgery alone) (5-year OS, $66.9 \%$ vs. $46.0 \%$ vs. $43.8 \%$; $\mathrm{P}=0.011$ ). Similar results were also reported by Ahmed et al., who observed the highest survival rate in stage IA SCLC patients who received surgery plus radiation $(60 \%)$, followed by surgery alone (50\%), radiation alone (27\%), and no surgery or radiation (16\%) (6). We attribute this to the unique biological characteristics of SCLC. Compared with NSCLC, SCLC is characterized by rapid doubling, high growth fraction, and the early development of widespread metastases $(21,26)$. Most patients with SCLC present with hematogenous metastases, approximately one-third present with limited disease confined to the chest, and fewer than $5 \%$ of patients have true stage I SCLC $(27,28)$. In this study, the 5-year OS for resected SCLC with stage I disease was significantly lower (48.9\%) than that of stage I NSCLC, but similar to that of stage IIIA NSCLC (15). These results suggest that SCLC is an aggressive disease even in the early stage, warranting multi-modality therapy. Further analysis after classification by resection type revealed that the efficacy of additional chemotherapy or radiotherapy on long-term survival was significant in the lobectomy cohort $(\mathrm{P}=0.012)$ but not in the sublobectomy cohort $(\mathrm{P}=0.752)$. As indicated by previous studies, postoperative chemo- radiotherapy might be beneficial to patients with resected lung cancer $(29,30)$. We speculate that the efficacy of surgery is limited for patients who undergo sublobectomy, and further restricts the efficacy of additional chemo/ radiotherapy. Similarly, Veluswamy et al. found adjuvant radiotherapy and chemotherapy was associated with increased toxicity and decreased survival in 1,929 NSCLC patients who underwent limited resection (31).

Extended lymphadenectomy with more lymph nodes examined has long been regarded as essential to accurate staging and long-term survival for operable lung cancer patients. This is also applicable to those with declared nodenegative disease $(32,33)$. In this study, the survival difference between ELN $\leq 7$ and ELN $>7$ was not significant based on PSM analysis $(\mathrm{P}=0.105)$. However, the absolute risk was reduced in patients with ELN $>7$ (crude cohort: HR $=0.709,95 \%$ CI, 0.511-0.984; matched cohort: HR $=0.755$, $95 \% \mathrm{CI}, 0.535-1.065$ ), the survival duration of $\mathrm{ELN}>7$ was longer than that of $\mathrm{ELN} \leq 7$ (crude cohort, 91 vs. 38 months; matched cohort, $91 v s .54$ months), and the superiority of more ELN scan be observed in survival curves. Therefore, extended lymphadenectomy with increased ELNs is still recommended for stage IA SCLC. In addition, the reduced hazard ratio of the ELN $>7$ group was similar to that in patients with oligo-modality therapy, suggesting that the impact of lymphadenectomy on long-term survival may be independent of perioperative CRT.

To date, improving the prognosis for SCLC patients has been the subject of numerous investigations. Some recent reports established the link between surgery and improved prognosis for early-stage SCLC. For example, Xu et al. 
found that stage IA SCLC patients who underwent surgery displayed a significantly longer median OS of 45 months, compared to those who did not receive surgery (20.0 months, $\mathrm{P}<0.001)$ (34). In addition, Yang et al. observed a higher 5 -year OS rates in SCLC patients with cT1-2N0M0 who received surgery than in those who underwent chemoradiation (47.6\% vs. $29.8 \%, \mathrm{P}<0.01$ ) (35). To address uncertainties surrounding the appropriate operation plan, $\mathrm{Gu} e t \mathrm{al}$. suggested that the prognosis of sublobectomy is comparable to that of lobectomy for IA stage SCLC (36). Our findings in this study further confirm the current understanding of early surgical intervention and its association with improved survival. In addition, this study demonstrates the benefits of additional chemo/radiation (median OS, 91 vs. 50 months; estimated 5-year OS, 54.0\% vs. $45.1 \%, \mathrm{P}<0.001)$ in resected, stage IA SCLC patients. The most promising prognosis was observed in patients who received lobectomy plus CRT (median OS, unreached; estimated 5-year OS, 73.0\%). Therefore, we believe that our investigation holds special significance and provides supplementary information to the existing evidence on stage IA SCLC.

According to our results, we recommend regular positron emission tomography/computed tomography (PET/CT) scanning during preoperative examination prior to lobectomy plus CRT for stage IA SCLC patients. In addition, more studies are needed to uncover other surgeryrelated prognostic factors beyond traditional staging, such as the required scope of lymphadenectomy and the minimal number of ELNs. The impact of emerging prognostic factors in NSCLC, such as "GGN (ground glass nodule) ratio" and "standardized uptake value in PET", also need to be clarified in the SCLC population $(37,38)$. Incorporating these factors will be vital in developing future guidelines for application into clinical practice and understanding the greater policy implications.

The underlying mechanism of our results is still unclear. However, we speculate that this might be partly attributable to the aggressive biological behavior of SCLC. For stage IA SCLC, although the disease is limited and surgery is able to achieve better outcome than CRT. However, the biological characteristics including aggressive invasion and early metastasis made greater margin distance an essential condition for operable SCLC. Besides, because SCLC is sensitive to chemo/radiotherapy, therefore operative chemo/radiotherapy is associated with improved outcome in this cohort.

This retrospective study utilized the SEER database for patient selection. Heterogeneities, including those associated with the basic characteristics of patients involving race, education, and income or the determination of treatment, were unavoidable. In addition, the sample size of each treatment modality was limited, possibly leading to bias in the analysis. Further validation from multicenter, prospective studies are warranted to overcome the limitations associated with the population size, heterogeneity, and potential study bias.

\section{Conclusions}

In conclusion, lobectomy plus CRT treatment is associated with longer survival and should be considered as part of the clinical management of stage IA SCLC patients.

\section{Acknowledgments}

Funding: This work was supported by grants from the Medical Scientific Research Foundation of Guangdong Province of China (Grant No. A2017327, A2018301), and the Health and Family Planning Commission of Shenzhen Municipality Research Project (Grant No. SZBC2018018).

\section{Footnote}

Reporting Checklist: The authors have completed the STROBE reporting checklist. Available at http://dx.doi. org/10.21037/atm-20-5525

Conflicts of Interest: All authors have completed the ICMJE uniform disclosure form (available at http://dx.doi. org/10.21037/atm-20-5525). The authors have no conflicts of interest to declare.

Ethical Statement: The authors are accountable for all aspects of the work in ensuring that questions related to the accuracy or integrity of any part of the work are appropriately investigated and resolved. The study was conducted in accordance with the Declaration of Helsinki (as revised in 2013).

Open Access Statement: This is an Open Access article distributed in accordance with the Creative Commons Attribution-NonCommercial-NoDerivs 4.0 International License (CC BY-NC-ND 4.0), which permits the noncommercial replication and distribution of the article with the strict proviso that no changes or edits are made and the 
original work is properly cited (including links to both the formal publication through the relevant DOI and the license). See: https://creativecommons.org/licenses/by-nc-nd/4.0/.

\section{References}

1. Bray F, Ferlay J, Soerjomataram I, et al. Global cancer statistics 2018: GLOBOCAN estimates of incidence and mortality worldwide for 36 cancers in 185 countries. CA Cancer J Clin 2018;68:394-424.

2. Bernhardt EB, Jalal SI. Small Cell Lung Cancer. Cancer Treat Res 2016;170:301-22.

3. Nesbit EG, Leal TA, Kruser TJ. What is the role of radiotherapy for extensive-stage small cell lung cancer in the immunotherapy era? Transl Lung Cancer Res 2019;8:S153-62.

4. Jett JR, Schild SE, Kesler KA, et al. Treatment of small cell lung cancer: Diagnosis and management of lung cancer, 3rd ed: American College of Chest Physicians evidencebased clinical practice guidelines. Chest 2013;143:e400S19S.

5. Weksler B, Nason KS, Shende M, et al. Surgical resection should be considered for stage I and II small cell carcinoma of the lung. Ann Thorac Surg 2012;94:889-93.

6. Ahmed Z, Kujtan L, Kennedy KF, et al. Disparities in the Management of Patients With Stage I Small Cell Lung Carcinoma (SCLC): A Surveillance, Epidemiology and End Results (SEER) Analysis. Clin Lung Cancer 2017;18:e315-25.

7. Varlotto JM, Recht A, Flickinger JC, et al. Lobectomy leads to optimal survival in early-stage small cell lung cancer: a retrospective analysis. J Thorac Cardiovasc Surg 2011;142:538-46.

8. Iwata T, Nishiyama N, Nagano K, et al. Role of pulmonary resection in the diagnosis and treatment of limited-stage small cell lung cancer: revision of clinical diagnosis based on findings of resected specimen and its influence on survival. Gen Thorac Cardiovasc Surg 2012;60:43-52.

9. Resio BJ, Hoag J, Chiu A, et al. Prophylactic cranial irradiation is associated with improved survival following resection for limited stage small cell lung cancer. J Thorac Dis 2019;11:811-8.

10. Liu J, Cui F, Pompeo E, et al. The impact of nonintubated versus intubated anaesthesia on early outcomes of video-assisted thoracoscopic anatomical resection in non-small-cell lung cancer: a propensity score matching analysis. Eur J Cardiothorac Surg 2016;50:920-5.

11. Liang W, Zhang L, Jiang G et al. Development and validation of a nomogram for predicting survival in patients with resected non-small-cell lung cancer. J Clin Oncol 2015;33:861-9.

12. Sagawa M, Oizumi H, Suzuki H, et al. A prospective 5 -year follow-up study after limited resection for lung cancer with ground-glass opacity. Eur J Cardiothorac Surg 2018;53:849-56.

13. Fiorelli A, Mauro I, Cicchitto G, et al. Lobar or sublobar resections are safe procedures for management of early lung cancer. Ann Transl Med 2019;7:S107.

14. Millum J, Wendler D, Emanuel EJ. The 50th anniversary of the Declaration of Helsinki: progress but many remaining challenges. JAMA 2013;310:2143-4.

15. Detterbeck FC, Boffa DJ, Kim AW, et al. The Eighth Edition Lung Cancer Stage Classification. Chest 2017;151:193-203.

16. D'Agostino, R. B., Jr. Propensity score methods for bias reduction in the comparison of a treatment to a nonrandomized control group. Stat Med 1998;17:2265-81.

17. Rosenbaum P, Rubin D. Constructing a control group using multivariate matched sampling methods that incorporate the propensity score. Am Stat 1985;39:33-8.

18. Le Ray I, Dabakuyo S, Crehange G, et al. Neoadjuvant therapy for breast cancer has no benefits on overall survival or on the mastectomy rate in routine clinical practice. A population-based study with a median follow-up of 11 years using propensity score matching. Eur J Cancer 2012;48:2300-10.

19. Wang F, Zheng $Y$, Wang $Z$, et al. Nodal skip metastasis in esophageal squamous cell carcinoma underwent three-field lymphadenectomy. Ann Thorac Surg 2017;104:1187-93.

20. Zeng H, Liu Y, Xia X, et al. Prognostic Analysis of Lobectomy versus Sublobar Resection in Patients Aged $>/=60$ Years with Stage Ia Small Cell Lung Cancer. Zhongguo Fei Ai Za Zhi 2018;21:8-15.

21. Schneider BJ, Saxena A, Downey RJ. Surgery for earlystage small cell lung cancer. J Natl Compr Canc Netw 2011;9:1132-9.

22. Goldstein NS, Ferkowicz M, Kestin L, et al. Wedge resection margin distances and residual adenocarcinoma in lobectomy specimens. Am J Clin Pathol 2003;120:720-4.

23. Dai C, Shen J, Ren Y, et al. Choice of Surgical Procedure for Patients With Non-Small-Cell Lung Cancer $</=1 \mathrm{~cm}$ or $>1$ to $2 \mathrm{~cm}$ Among Lobectomy, Segmentectomy, and Wedge Resection: A Population-Based Study. J Clin Oncol 2016;34:3175-82.

24. Mei J, Guo C, Xia L, et al. Long-term survival outcomes of video-assisted thoracic surgery lobectomy for stage 
I-II non-small cell lung cancer are more favorable than thoracotomy: a propensity score-matched analysis from a high-volume center in China. Transl Lung Cancer Res 2019;8:155-66.

25. Zhao ZR, Situ DR, Lau RWH, et al.Comparison of Segmentectomy and Lobectomy in Stage IA Adenocarcinomas. J Thorac Oncol 2017;12:890-6.

26. Oliver DE, Donnelly OG, Grass GD, et al. Extracranial metastatic burden in extensive-stage small cell lung cancer: implications for prophylactic cranial irradiation. J Thorac Dis 2018;10:4321-7.

27. Kinoshita T, Yoshida J, Ishii G, et al. The differences of biological behavior based on the clinicopathological data between resectable large-cell neuroendocrine carcinoma and small-cell lung carcinoma. Clin Lung Cancer 2013;14:535-40.

28. Ignatius Ou SH, Zell JA. The applicability of the proposed IASLC staging revisions to small cell lung cancer (SCLC) with comparison to the current UICC 6th TNM Edition. J Thorac Oncol 2009;4:300-10.

29. Uy KL, Darling G, Xu W, et al. Improved results of induction chemoradiation before surgical intervention for selected patients with stage IIIA-N2 non-small cell lung cancer. J Thorac Cardiovasc Surg 2007;134:188-93.

30. Kim BH, Kim HJ, Wu HG, et al. Role of postoperative radiotherapy after curative resection and adjuvant chemotherapy for patients with pathological stage N2 non-small-cell lung cancer: a propensity score matching analysis. Clin Lung Cancer 2014;15:356-64.

31. Veluswamy RR, Mhango G, Bonomi M, et al. Adjuvant treatment for elderly patients with early-stage lung cancer treated with limited resection. Ann Am Thorac Soc 2013;10:622-8.

Cite this article as: Lin SF, Zheng YZ, Li XQ, Xu HP, Wang JJ, Wang W, Huang QY, Wu D, Zhong CX, Fu SS, Yuan LX, Wang SC, Luo RX, Zhai WY, Yu BT, Zhu KS. Impact of treatment modality on long-term survival of stage IA small-cell lung cancer patients: a cohort study of the U.S. SEER database. Ann Transl Med 2020;8(20):1292. doi: 10.21037/atm-205525
32. Liang W, He J, Shen Y, et al. Impact of Examined Lymph Node Count on Precise Staging and Long-Term Survival of Resected Non-Small-Cell Lung Cancer: A Population Study of the US SEER Database and a Chinese Multi-Institutional Registry. J Clin Oncol 2017;35:1162-70.

33. Lim E, Belcher E, Yap YK, et al. The role of surgery in the treatment of limited disease small cell lung cancer: time to reevaluate. J Thorac Oncol 2008;3:1267-71.

34. Xu L, Zhang G, Song S, et al. Surgery for small cell lung cancer: A Surveillance, Epidemiology, and End Results (SEER) Survey from 2010 to 2015. Medicine (Baltimore) 2019;98:e17214.

35. Yang CJ, Chan DY, Shah SA, et al. Long-term Survival After Surgery Compared With Concurrent Chemoradiation for Node-negative Small Cell Lung Cancer. Ann Surg 2018;268:1105-12.

36. Gu C, Huang Z, Dai C, Wang Y, et al. Prognostic Analysis of Limited Resection Versus Lobectomy in Stage IA Small Cell Lung Cancer Patients Based on the Surveillance, Epidemiology, and End Results Registry Database. Front Genet 2018;9:568.

37. Hattori A, Matsunaga T, Takamochi K, et al. Indications for sublobar resection of clinical stage IA radiologic puresolid lung adenocarcinoma. J Thorac Cardiovasc Surg 2017;154:1100-8.

38. Kodama K, Higashiyama M, Okami J, et al. Oncologic Outcomes of Segmentectomy Versus Lobectomy for Clinical T1a N0 M0 Non-Small Cell Lung Cancer. Ann Thorac Surg 2016;101:504-11.

(English Language Editors: E. Tan and J. Gray) 
Supplementary

Table S1 Treatment characteristics in patients with resected SCLC

\begin{tabular}{lc}
\hline Variables & Case (\%) \\
\hline Total & 337 \\
Surgical resection & \\
Lobectomy & $247(73.3)$ \\
Sublobectomy & $90(26.7)$ \\
Examined lymph nodes & \\
$\leq 7$ & $185(54.9)$ \\
$>7$ & $152(45.1)$ \\
Chemotherapy & \\
Yes & $189(56.1)$ \\
No/unknown & $148(43.9)$ \\
Radiotherapy & \\
Yes & $74(22.0)$ \\
No/unknown & $263(78.0)$ \\
Treatment modality & $146(21.4)$ \\
Oligo-modality & \\
Bi-modality & \\
Tri-modality & \\
\hline
\end{tabular}

SCLC, small cell lung cancer.

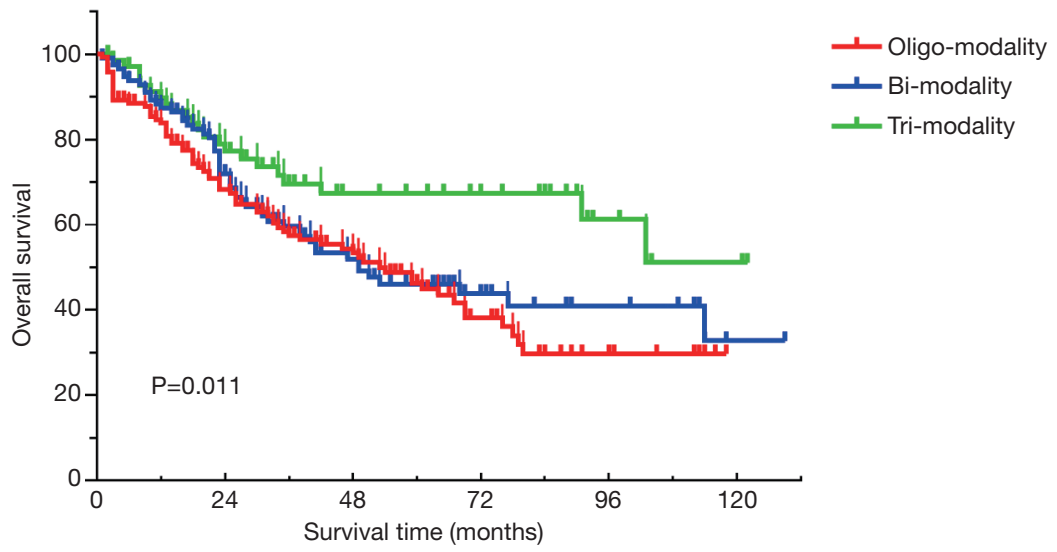

Figure S1 Comparison among oligo-modality (surgery alone), bi-modality (surgery plus chemo/radiotherapy), and tri-modality (surgery plus chemoradiotherapy) in patients with resected SCLC. SCLC, small cell lung cancer. 